\title{
Cigarrillo electrónico y otros sistemas electrónicos de liberación de nicotina: revisión de evidencias sobre un tema controversial
}

\author{
Laura Llambí*, Diego Rodríguez ${ }^{\dagger}$, Carolina Parodił, Enrique Soto§
}

\section{Resumen}

Introducción: los cigarrillos electrónicos (CE) son sistemas que liberan aerosol conteniendo nicotina y otras sustancias. Desde su aparición han generado una gran controversia en la comunidad científica dedicada al control de tabaco, existiendo evidencias que los posicionan como una alternativa de tratamiento para la cesación tabáquica y otros estudios que muestran sus daños a la salud, así como convertirlos en la puerta de entrada a los jóvenes a la adicción a nicotina.

Objetivo: recopilar evidencia disponible sobre CE con referencia a componentes, riesgos para la salud, cesación de tabaquismo, impacto poblacional y aspectos normativos y regulatorios.

Método: revisión narrativa. Se realizó búsqueda bibliográfica en bases PubMed, MEDLINE, SCOPUS, Redalyc, SciELO, LiLACS, Dialnet, ScienceDirect, así como en documentos de la Organización Mundial de la Salud, sociedades científicas, sitios web dedicados a consumidores y legislación nacional e internacional.

Resultados: se revisaron 130 documentos, de los cuales se toman los 63 más relevantes. Existen evidencias científicas sobre contenidos tóxicos y carcinógenos en líquidos y aerosoles de los $\mathrm{CE}$, así como de daño a la salud cardiovascular y respiratoria. Se presentan estudios controversiales sobre su eficacia para dejar de fumar. Se presenta evidencia acerca de cómo los CE impactan negativamente en la iniciación de jóvenes al consumo y en la recaída de exfumadores. Se analiza la normativa nacional e internacional para regular los $\mathrm{CE}$.

Conclusiones: los profesionales de la salud no deben aconsejar el uso de $\mathrm{CE}$, dado que no son seguros y exponen a los consumidores y a la población en general de los efectos deletéreos directos e indirectos de los $\mathrm{CE}$.

Palabras clave: Cigarrillo electrónico

Vapeo

Sistemas electrónicos de liberación de nicotina

Tabaquismo

Cese del hábito de fumar

Key words: $\quad$ Electronic cigarette

Vaping

Electronic nicotine delivery systems

Tobacco use disorder

Smoking cessation

\footnotetext{
* Médica Internista Unidad de Tabaquismo, Clínica Médica "A" Hospital de Clínicas, Facultad de Medicina, Universidad de la República. † Sociólogo. Centro de Investigación para la Epidemia del Tabaquismo.

‡ Médica Internista Unidad de Tabaquismo, Clínica Médica "A" Hospital de Clínicas, Facultad de Medicina,Universidad de la República.

$\S$ Médico Cardiólogo. Programa Nacional para el Control de Tabaco. Ministerio de Salud.

Los autores declaran no tener conflicto de intereses

Correspondencia: Dra. Laura Llambí. Hospital de Clínicas. Avda. Italia s/n esq. Las Heras, piso 11. CP 11600 Montevideo, Uruguay. Correo elec-

trónico: llambil@hc.edu.uy laura.llambi@gmail.com

Recibido: $17 / 6 / 19$

Aprobado: $5 / 8 / 19$
} 


\section{Introducción}

Los cigarrillos electrónicos (CE), e-cigarettes y otros sistemas electrónicos de administración de nicotina (SEAN), sin nicotina (SSSC) o vaporizadores de nicotina son sistemas que liberan aerosol con sustancias que puede contener nicotina ${ }^{(1,2)}$. El CE fue creado en China en el año 2003 y patentado en el 2007. Incluso antes de ser patentado, las ventas habían alcanzado a Europa y Estados Unidos, y desde entonces se ha asistido a un rápido crecimiento en el marketing, ventas y utilización que también ha llegado a Uruguay. En nuestro país, en 2014, la Encuesta ITC (Proyecto Internacional de Evaluación de Políticas de Control del Tabaco) ${ }^{(3)}$ mostró que una tercera parte de los fumadores había oído hablar de los CE, y de ellos 8\% los había probado. La mayoría $(71 \%)$ creía que eran menos dañinos, la cuarta parte $(25 \%)$ creía que eran igual de perjudiciales y $4 \%$ que lo eran más que el cigarrillo. En un estudio realizado en el Hospital de Clínicas en 2015, con estudiantes de Medicina, $72,2 \%$ de los encuestados conocía el CE, mientras que 7,5\% lo había utilizado. Entre los más de 300 estudiantes encuestados, $41,5 \%$ creía que los CE eran dañinos y $44,4 \%$ no tenía conocimiento acerca de sus potenciales daños. Más de una cuarta parte de los estudiantes desconocía que la venta de estos dispositivos está prohibida en Uruguay ${ }^{(4)}$. La Encuesta Global de Tabaco en Adultos realizada en Uruguay en 2017 recogió números similares: $45 \%$ de los encuestados alguna vez oyó hablar de CE, solo 3,1\% los había probado y $0,2 \%$ eran usuarios de CE. El $43 \%$ considera que son menos perjudiciales que el cigarrillo convencional ${ }^{(5)}$.

Desde su aparición, los dispositivos han sido mejorados en su tecnología, existiendo diversas marcas, tipos, formas y tamaños, que hacen más atractivo el producto y se han "esponsorizado", alentado en su uso como dispositivos menos dañinos para la salud.

Desde 2014, la industria tabacalera ha invertido en el mercado de los CE, incluida la compañía china original Ruyan, que fue comprada por Imperial Tobacco.

A pesar de contener sustancias de las que se conoce su potencial daño y otras aún en estudio, muchos fumadores globalmente lo utilizan con creciente frecuencia y la comunidad científica mundial se encuentra en controversia con el tema, existiendo un número de investigadores que propenden su uso como alternativa en la cesación.

El objetivo de este trabajo es recopilar y analizar la evidencia disponible sobre $\mathrm{CE}$ y otros dispositivos con referencia a sus componentes, riesgos para la salud, la contribución en la cesación de tabaquismo, impacto poblacional de éstos en las tendencias de consumo de tabaco así como revisar aspectos normativos y regulatorios.

\section{Material y método}

Se realizó una búsqueda sistemática en la literatura académica que incluyó revisiones y artículos de investigación sobre dispositivos electrónicos de liberación de nicotina sin límite temporal y hasta abril de 2019. Los autores, de forma independiente, realizaron una búsqueda en los principales portales de publicaciones académicas o repositorios de publicaciones, que incluyó: PubMed, MEDLINE, SCOPUS, Redalyc, SciELO, LiLACS, Dialnet, Science Direct. Para la búsqueda se utilizaron los siguientes descriptores o palabras claves: "e-cigarrette", "cigarrillo electrónico", "Electronic Nicotine Delivery System". Asimismo, se revisaron los documentos emitidos por las Conferencias de las Partes (COP) en el Convenio Marco (CMCT) de la Organización Mundial de la Salud (OMS) para el Control del Tabaco, documentos de posición de sociedades científicas, sitios dedicados a consumidores, así como la normativa nacional en la materia. Se revisaron 130 artículos de los cuales se seleccionaron los 63 más relevantes.

\section{Tipos de dispositivos y características}

En el mercado coexisten varios tipos de dispositivos con diferencias potenciales en la producción de sustancias tóxicas y la administración de nicotina: los de primera generación o CE similares a sus pares convencionales de tabaco (cig-a-likes), los sistemas de tanque de segunda generación, e incluso vaporizadores personales más grandes o de tercera generación ${ }^{(2,6)}$. De acuerdo con otras clasificaciones, estos dispositivos se dividen en sistemas abiertos y cerrados, dependiendo fundamentalmente del grado de control que los usuarios tienen sobre la solución líquida y el voltaje y resistencia aplicados para calentarla, así como las características de ventilación. Algunos $\mathrm{CE}$ se asemejan a cigarrillos, cigarros o pipas; otros parecen lapiceras, pendrives, entre otros formatos (figuras 1 a 3$)^{(1,2)}$.

Constan de tres partes que se ensamblan: la batería, el atomizador y el cartucho. En la mayoría de los dispositivos el cartucho es recargable, aunque también se puede cambiar por otro. Cada cartucho equivale a unas 300 caladas, lo que representa unos 20 cigarrillos, pero al depender en forma individual de la intensidad y frecuencia de la inhalación, resulta difícil saber la cantidad de nicotina que se puede llegar a consumir ${ }^{(7)}$.

Existen distintas marcas de CE, entre ellas, se puede mencionar: Vaporesso, Hangsen, Eleaf, Joyetech, Kanger, Innokin, Smok, Aspire, Vaptio, Suorin, Justfog, Digiflavor, Vype, Juul, Blu. El caso de Juul, uno de los CE con mayor venta en Estados Unidos, es una marca vinculada a la compañía Philip Morris. Fue lanzado al mercado en 2015 y es similar a un pendrive (figura 4). Está 


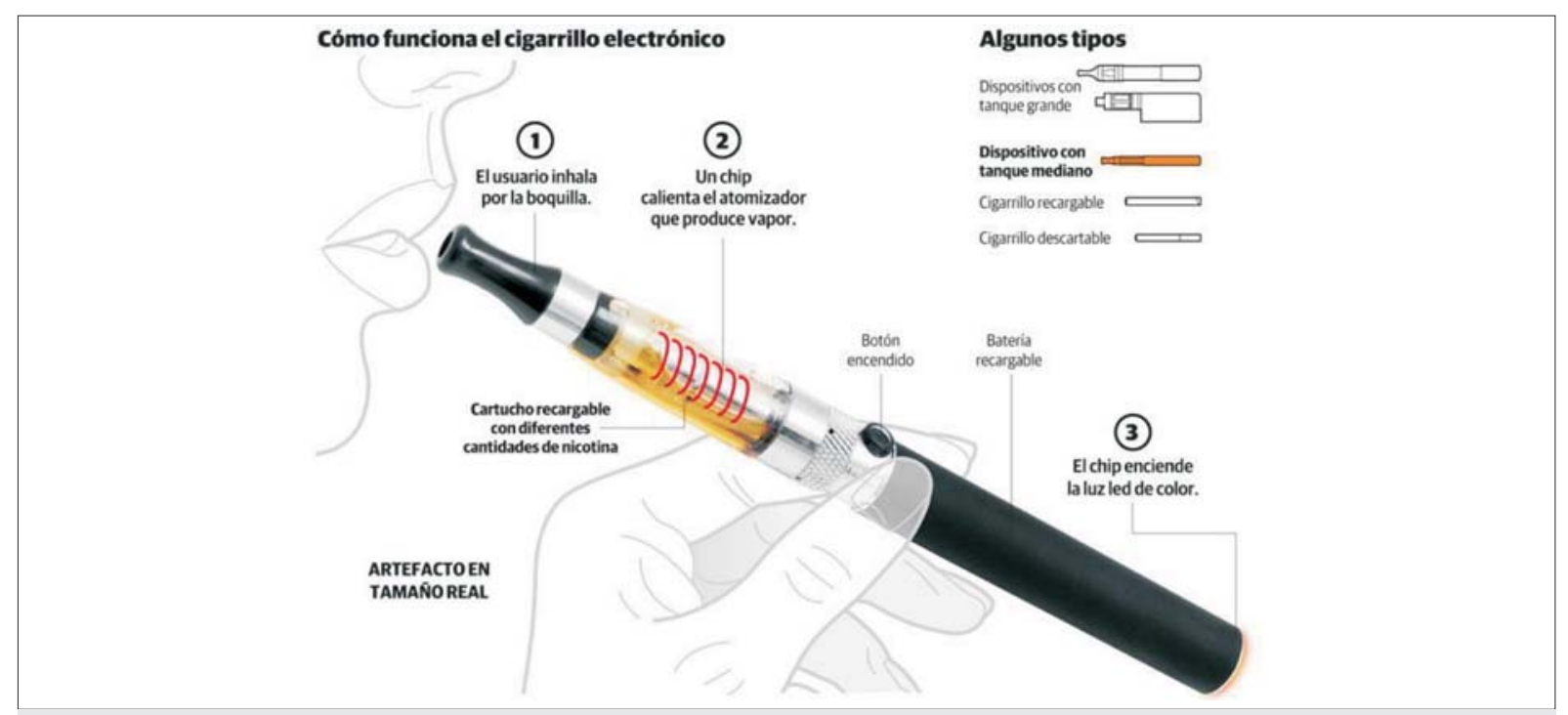

Figura 1. Cigarrillo electrónico con tanque mediano.

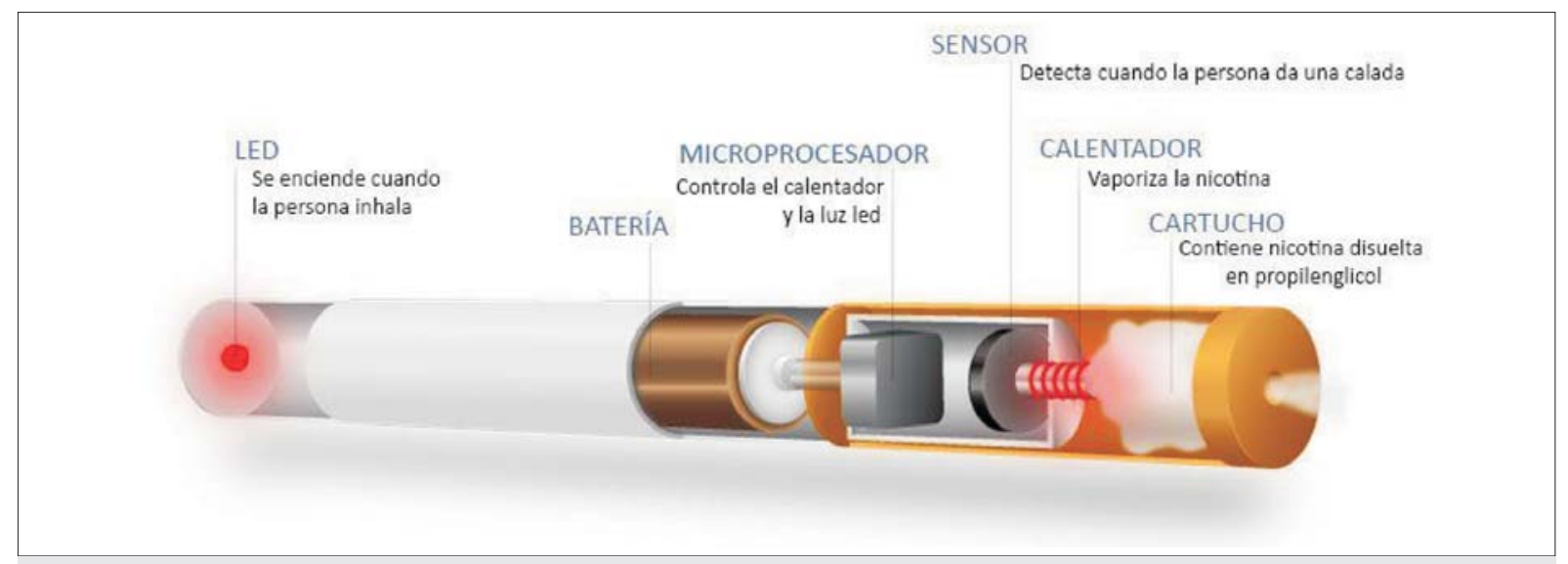

Figura 2. Composición del cigarrillo electrónico.

compuesto por el dispositivo (cuerpo principal) y una cápsula que se inserta. Además, cuenta con un conector USB para poder recargar el dispositivo en cualquier puerto de ese tipo. JUUL nació como un proyecto de la estadounidense PAX Labs, convirtiéndose en 2017 en el CE más popular de Estados Unidos. Actualmente, Philip Morris, una de las mayores empresas tabacaleras, ha invertido fuertemente en la empresa fabricante ${ }^{(8)}$.

A quienes consumen este tipo de productos, se los denomina o se autodenominan "vapeadores", nombre originado porque el dispositivo desprende vapor. La cantidad de vapor que desprenden es una de las características que se tienen en cuenta al momento de adquirir el producto, de acuerdo a los sitios web disponibles dedicados a estos consumidores ${ }^{(9)}$. La elección también puede estar vinculada a si se asemeja a un cigarrillo tradicio- nal o si es de diseño novedoso. El primer tipo (figura 5) sería escogido por quienes se inician en CE, prefieren algo más convencional y económico. Los consumidores perciben su desventaja en que emiten poco vapor ${ }^{(9)}$. Los dispositivos con diseños novedosos son valorados por la mayor densidad y cantidad de vapor emitida, aunque se considera una desventaja su mayor tamaño, mayor consumo de líquido y su costo.

\section{Contenidos y emisiones de la sustancia vaporizada}

La solución que contienen está compuesta por diferentes sustancias y saborizantes, por lo general, entre ellas, se incluye al propilenglicol, glicerina, saborizantes y usualmente hasta $36 \mathrm{mg}$ de nicotina. En algunos casos se han detectado hasta $100 \mathrm{mg}$ de nicotina ${ }^{(10-12)}$. 


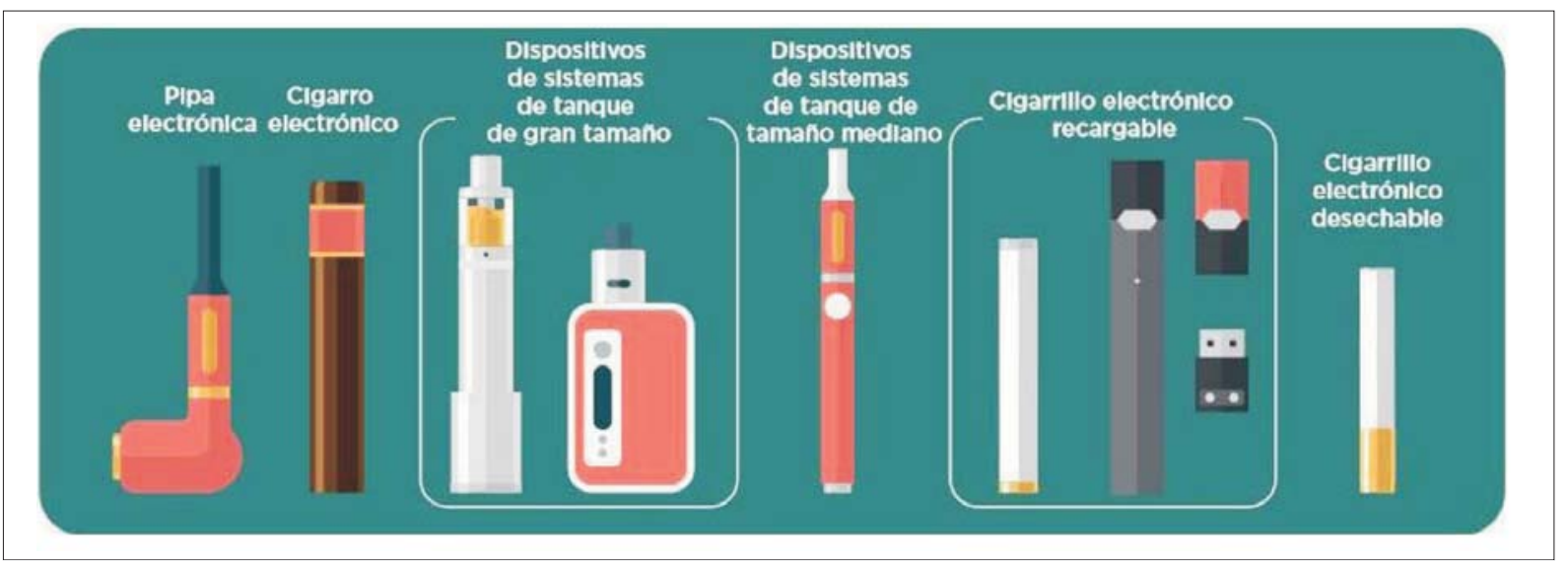

Figura 3. Distintos tipos de cigarrillos electrónicos.

También se ha encontrado que contienen sustancias como formaldehído, acetaldehído, acroleína y distintos metales pesados. Algunas de estas sustancias son catalogadas como citotóxicas y carcinógenas. También se ha observado que algunos CE que se comercializan como productos sin nicotina, en realidad sí la tienen ${ }^{(1,2,7,13,14)}$.

El propilenglicol es un compuesto presente en todos los dispositivos, también presente en alimentos, cosméticos, inhaladores y en la "niebla artificial" utilizada en teatros y espectáculos musicales. Irrita las mucosas de las vías respiratorias al ser inhalado ${ }^{(15)}$. Una dosis superior a $309 \mathrm{mg} / \mathrm{m}^{3}$ durante más de un minuto puede irritar ojos, orofaringe y vías respiratorias. La glicerina se considera segura cuando es consumida por vía oral, pero se desconocen sus efectos cuando es inhalada ${ }^{(10)}$. Los dicarbonilos (glioxal, metilglioxal, diacetilo) e hidroxicarbonilos (acetol) se consideran compuestos importantes del aerosol generado por estos dispositivos. Muchos de estos componentes son sustancias tóxicas, con efectos conocidos sobre la salud, que producen cambios patológicos significativos ${ }^{(14,15)}$.

Con la intención de hacer más atractivos los productos, se han introducido sustancias que al ser calentadas producen aromas. Se han registrado cerca de 8.000 aromas en las soluciones líquidas de los sistemas electrónicos. Algunas de ellas podrían incrementar los efectos inflamatorios sobre las mucosas del sistema respiratorio, otros, como los aromatizantes de maíz tostado, canela y cereza, son considerados potencialmente peligrosos para la salud.

Cuando el consumidor vapea (inhala), se activa un mecanismo que, sin mediar combustión, calienta la solución generando un aerosol que el consumidor inhala y luego es exhalado o se despide en forma de vapor ${ }^{(16)}$. Este vapor permanece en el ambiente y sus contenidos pueden ser inhalados de forma pasiva. El aerosol ajeno es una nueva fuente de contaminación del aire por partí- culas, entre las que se incluyen las partículas finas y ultrafinas, así como propanediol, ciertos compuestos orgánicos volátiles, metales pesados y nicotina ${ }^{(17)}$. En cuanto a los metales pesados encontrados en un estudio, se hallaron partículas metálicas de $1 \mathrm{~mm}$ compuestas de estaño, plata, hierro, níquel, aluminio y silicato, y nanopartículas $(100 \mathrm{~nm})$ de estaño, cromo y níquel. Las concentraciones de nueve de once elementos analizados en el aerosol fueron mayores o iguales a las correspondientes a un cigarrillo convencional ${ }^{(18)}$.

\section{Daños a la salud}

Los CE podrían ser menos dañinos que los cigarrillos regulares, lo que no significa que sean seguros. Si bien el aerosol producido contiene menos sustancias químicas tóxicas que los cigarrillos regulares, estas sustancias no están exentas de perjuicios para la salud de sus consumidores. Se debe tener en cuenta que la nicotina es una sustancia potente adictiva, que puede ser tóxica en contacto directo con la piel o que puede ser mortal si se ingiere en una dosis superior a los $6 \mathrm{mg}$, lo cual representa un riesgo elevado para los niños que puedan acceder al producto ante un descuido de sus padres ${ }^{(10)}$.

\section{Daños sobre el aparato cardiovascular}

Las evidencias de daño de los CE sobre la salud cardiovascular surgen desde modelos experimentales hasta estudios en humanos con resultados "duros", como la ocurrencia de eventos. En modelos animales y cultivos celulares ya se ha establecido que las emisiones y vapor de los CE activan pasos del estrés oxidativo y disfunción endotelial, que son los precursores de la enfermedad preclínica ${ }^{(19)}$. La utilización de CE ha impactado en la salud cardiovascular, según un estudio basado en registros de salud en Estados Unidos comprendidos entre los años 2014 y 2017. El estudio concluye que los consumidores de los mencionados dispositivos están más 


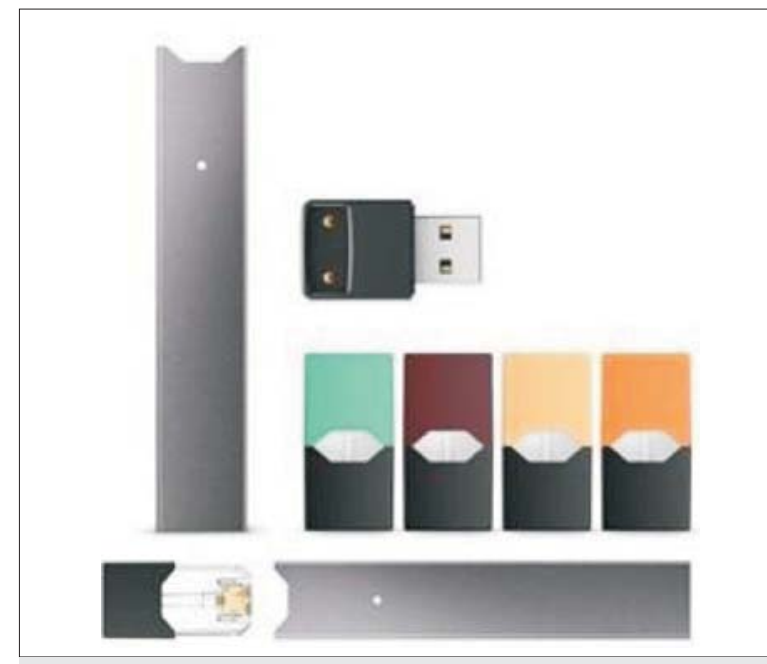

Figura 4. Cigarrillo electrónico JUUL.

expuestos a padecer infarto agudo de miocardio y enfermedades cerebrovasculares ${ }^{(20,21)}$.

\section{Daño sobre el aparato respiratorio}

El consumo de CE se ha asociado al aumento de la resistencia periférica de la vía aérea y de la fracción de óxido nítrico exhalado, con similares características a los efectos del humo de tabaco ${ }^{(22)}$. En un estudio reciente sobre 28.771 pacientes realizado en Estados Unidos, donde se compararon los síntomas respiratorios en fumadores, vapeadores y fumadores duales, se concluyó que los vapeadores tenían ostensiblemente más aumento de sibilancias que los no vapeadores, y que no existía diferencia entre fumadores y fumadores duales ${ }^{(23)}$. Las reiteradas exposiciones a acroleínas provenientes del calentamiento de propiletilenglicol y la glicerina causan inflamación crónica y disminución de las defensas con daño mediado por proteasas, lo que conllevaría al daño estructural, como el de la enfermedad pulmonar obstructiva crónica ${ }^{(24)}$.

Asimismo, la inmunosupresión en la mucosa nasal propendería a mayor riesgo de desarrollo de infecciones a Streptococcus pneumoniae y también ha habido reportes de casos de neumonía lipoidea ${ }^{(25)}$. Se ha encontrado el doble de casos de bronquitis en estudiantes de secundaria de Estados Unidos consumidores de CE y aumento de casos de asma en Corea entre los mismos ${ }^{(26)}$.

\section{Otros daños}

En abril de 2019, la Food and Drug Administration (FDA) lanzó una advertencia a los consumidores de CE sobre la posibilidad de éstos de producir convulsiones, basado en el incremento de reportes en los últimos años, y poniendo énfasis en el fácil acceso de los jóvenes a altos niveles de nicotina con un consumo más accesible y

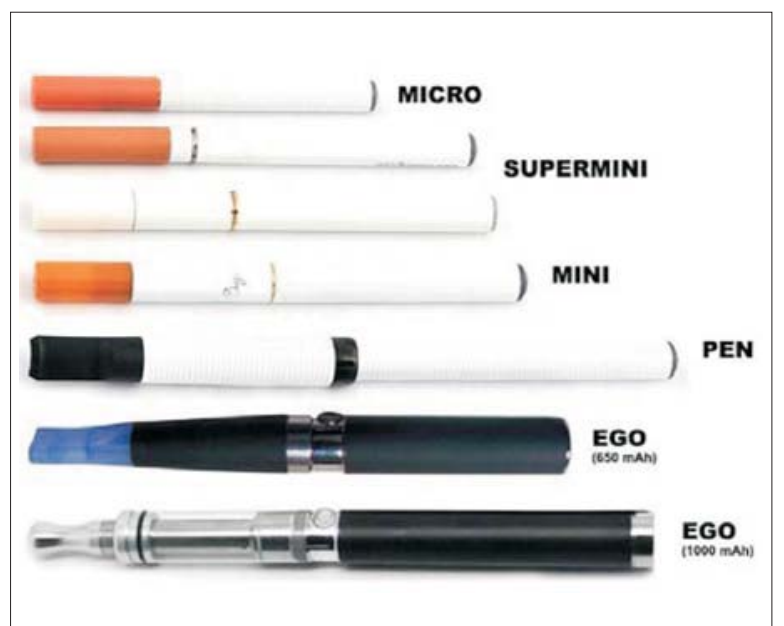

Figura 5. Algunas presentaciones de los cigarrillos electrónicos.

permisivo. También se han reportado explosiones y quemaduras por malfuncionamiento ${ }^{(27,28)}$.

Vapor de segunda mano. También hay resultados contradictorios respecto a si el inhalar en forma pasiva todos estos compuestos contenidos en el vapor ajeno constituye un daño a la salud de terceros. Mientras que algunos investigadores sostienen que las concentraciones de compuestos son inocuas para quienes se exponen $^{(29)}$, otros sostienen lo contrario. Mediciones de materia particulada en el aire en hogares donde se vapea, mostraron valores de partículas menores a 2,5 micras (con penetración alveolar pulmonar), similares a las halladas cuando se fuma tabaco en un hogar ${ }^{(17)}$ y superiores a las recomendadas por las agencias internacionales de calidad de aire ambiental. El volver a introducir en los ambientes libres de humo dispositivos que emiten vapor contribuye a renormalizar un hábito, disminuyendo los efectos de la política sanitaria.

\section{Evidencias relacionadas con las estrategias de cesación}

La gran controversia que han generado los CE en la comunidad científica del control de tabaco se explica con el surgimiento de evidencia de que estos dispositivos podrían ser efectivos para dejar de fumar. En 2013, Bullen y colaboradores publicaron un ensayo clínico aleatorizado (ECA) cuyo objetivo era comparar la efectividad de los $\mathrm{CE}$ versus la terapia de reemplazo nicotínico a forma de parches de $21 \mathrm{mg}$ o CE - placebo (sin nicotina) ${ }^{(30)}$. En este estudio de 13 semanas, en el que participaron 657 sujetos que deseaban dejar de fumar, se hallaron tasas de cesación relativamente pobres a los seis meses (7,3\% en la rama CE y 5,8\% en la rama parches), si se tiene en cuenta que la cesación espontánea sin nin- 
gún tipo de ayuda es de 3\% a 5\% cada año. El estudio no alcanzó un poder estadístico para probar superioridad de los CE frente a los parches de nicotina, pero luego de un análisis de no inferioridad post-hoc, los autores concluyen que el $\mathrm{CE}$ es al menos tan efectivo como la terapia de reemplazo nicotínico (TRN) para dejar de fumar. Otros resultados favorables que mostró este estudio fue un significativo descenso del número de cigarrillos fumados a diario en el grupo de CE que continuó fumando, un mayor tiempo libre de recaída y una adherencia superior al tratamiento cuando se compara con los parches de nicotina. Si bien no se reportan efectos adversos importantes, se destaca el corto período de seguimiento. En el mismo año, Caponetto y colaboradores realizaron el estudio ECLAT, que evaluó los CE como herramienta para reducción del consumo de tabaco y la abstinencia mantenida en fumadores sin intenciones de dejar de fumar ${ }^{(31)}$. Se trató de un ECA donde se comparaban dos ramas de $\mathrm{CE}$ con diferentes dosis de nicotina y una rama de CE sin nicotina. Observaron que los participantes de todas las ramas mostraron una tasa de cesación promedio de $10,7 \%$ a los tres meses y $8,3 \%$ al año. En aquellos que continuaron fumando, el consumo se redujo a más de la mitad en $22 \%$ a las 12 semanas y $10,3 \%$ al año, considerando las tres ramas en su conjunto. Las tasas son mayores aun en la rama de mayor dosis de nicotina en el dispositivo $^{(31)}$.

Estos dos estudios fueron metaanalizados en una revisión sistemática de Cochrane, en la que se concluye que los CE ayudan a dejar de fumar cuando se los compara con placebo, no estando claro su diferencia en efectividad respecto a los parches de nicotina. Los autores destacan que de acuerdo a los estándares GRADE la evidencia hasta el momento era de nivel bajo dado el escaso número de eventos (cesación), los intervalos de confianza amplios y la baja cantidad de estudios. Destacan que había varios estudios en curso que aclararían el rol de estos dispositivos ${ }^{(32)}$. En 2016, Tseng y colaboradores realizaron otro ensayo clínico en sujetos entre 21 y 35 años que no deseaban dejar de fumar. Los individuos fueron aleatorizados a utilizar CE con nicotina o CE-placebo. El estudio mostró una diferencia muy significativa con un valor $\mathrm{p}<0,001$ en la reducción del número de cigarrillos fumados por día en la rama $\mathrm{CE}$ con nicotina ${ }^{(33)}$. Los autores subrayan que el CE podría ser una herramienta útil en una etapa precesación en individuos poco motivados, logrando una disminución del consumo que podría aumentar las chances de éxito de un tratamiento convencional posterior. También destacan la preferencia de este método frente a otros por parte de los fumadores, ya que el mismo emula la conducta de fumar y reducen eficazmente los episodios de síndrome de abstinencia.
Otro estudio más pequeño, realizado en 2017 por Carpenter y colaboradores, en cambio, no logró demostrar que los CE fueran superiores a un grupo control en el logro de abstinencia, ni en el número de intentos de cesar. Nuevamente, sí se evidenció una reducción del número de cigarrillos al día en el grupo que usó $\mathrm{CE}^{(34)}$.

En 2018, Halpern y colaboradores realizaron un estudio que incluyó 6.006 fumadores sin tener en cuenta su motivación para dejar de fumar, cuyo objetivo era estudiar la efectividad de terapias farmacológicas, $\mathrm{CE}$ o incentivos económicos para dejar de fumar. El estudio tenía un enfoque pragmático, donde los sujetos no recibían la intervención, sino que se les ofrecía gratuitamente el acceso a ella cuando quisieran, intentando un escenario similar al de la vida real. Los individuos también recibieron información general y mensajes de texto motivacionales vía SMS, lo que se consideró cuidado habitual. El estudio encontró que los $\mathrm{CE}$ no fueron superiores al cuidado habitual $(\mathrm{p}=0,20)$, ni a la farmacoterapia $(p=0,43)^{(35)}$.

Más recientemente, Hayek y colaboradores publicaron otro estudio clínico aleatorizado que comparó nuevamente el uso de CE versus la TRN, ambos por tres meses, en adultos que asisten a los servicios de cesación de tabaquismo del Reino Unido ${ }^{(36)}$. Este estudio, a diferencia del realizado por Bullen en 2013, incluyó soporte cognitivo conductual a los pacientes de ambas ramas, y en el caso de la rama TRN, el propio usuario podía elegir la forma farmacéutica de preferencia entre parches, chicles, caramelos o combinaciones de éstos. La tasa de abstinencia, además, fue medida a los 12 meses. Se incluyeron 886 participantes, encontrándose 18\% de abstinencia al año en el grupo EC y $9,9 \%$ en la rama TRN (RR1,83 IC95\% 1,30-2,58; $\mathrm{p}<0,001$ ). El grupo EC mostró más efectos colaterales, como irritación orofaringe, mientras que el grupo TRN evidenció más náuseas, no encontrándose diferencias significativas en cuanto a la ocurrencia de sibilancias o disnea. El grupo EC mostró mayores tasas de adherencia al método propuesto. Los autores concluyen que los CE son más efectivos que la TRN para dejar de fumar cuando ambas estrategias se combinan con apoyo psicoconductual. Existen otros estudios en curso donde se combina $\mathrm{CE}$ con vareniclina o con TRN, cuyos resultados aún no están disponibles ${ }^{(37,38)}$. Un resumen de los estudios clínicos de CE se muestra en la tabla 1.

Con la controversia sobre la mesa, la Conferencia de las Partes para la implementación del Convenio Marco para el Control del Tabaco ha sugerido en sus sucesivas reuniones, y en la última realizada en el año 2018 en Ginebra, continuar con los estudios al respecto a los efectos de arribar a una conclusión con mayor evidencia $^{(39)}$. 
Tabla 1. Principales estudios clínicos aleatorizados acerca del cigarrillo electrónico.

\begin{tabular}{|c|c|c|c|c|c|}
\hline Autor, año & Población & Diseño de estudio & Tiempo seguimiento & Resultado buscado & $\begin{array}{l}\text { Interpretación de } \\
\text { hallazgos }\end{array}$ \\
\hline Bullen, 2013 & $\begin{array}{l}657 \text { individuos } \\
\text { motivados para dejar } \\
\text { de fumar }\end{array}$ & $\begin{array}{l}\text { ECA, no inferioridad } \\
3 \text { ramas, } 12 \text { semanas } \\
\text { - CE con nicotina } \\
\text { - CE sin nicotina } \\
\text { - Parche de nicotina } 21 \\
\text { mg }\end{array}$ & 6 meses & $\begin{array}{l}\text { Abstinencia continua a } \\
\text { los } 6 \text { meses verificada } \\
\text { por CO } \\
\text { Reducción del } \\
\text { consumo }\end{array}$ & $\begin{array}{l}\text { CE fue tan efectivo } \\
\text { como parches para } \\
\text { dejar de fumar } \\
\text { CE disminuye } \\
\text { consumo de cpd en } \\
\text { quienes siguen } \\
\text { fumando }\end{array}$ \\
\hline Caponetto, 2013 & $\begin{array}{l}300 \text { individuos no } \\
\text { motivados para dejar } \\
\text { de fumar }\end{array}$ & $\begin{array}{l}\text { ECA } \\
3 \text { ramas, } 12 \text { semanas } \\
\text { - CE con nicotina } 7,2 \\
\text { mg } \\
\text { - CE con nicotina } 7,2 \text { / } \\
5,4 \text { mg } \\
\text { - CE sin nicotina }\end{array}$ & 12 meses & $\begin{array}{l}\text { Reducción del } \\
\text { consumo al } 50 \% \\
\text { Abstinencia continua a } \\
12 \text { y } 52 \text { semanas } \\
\text { verificada por CO }\end{array}$ & $\begin{array}{l}\text { Las tres ramas } \\
\text { disminuyeron } \\
\text { significativamente los } \\
\text { cpd } \\
\text { Las ramas de CE con } \\
\text { mayor o menor dosis } \\
\text { de nicotina lograron } \\
\text { abstinencia a } 12 \\
\text { meses de } 13 \% \text { y } 9 \% \\
\text { respectivamente }\end{array}$ \\
\hline Tseng, 2016 & $\begin{array}{l}99 \text { adultos jóvenes ( } 21 \\
\text { y } 35 \text { años) no } \\
\text { motivados para dejar } \\
\text { de fumar }\end{array}$ & $\begin{array}{l}\text { ECA } \\
2 \text { ramas, } 3 \text { semanas } \\
- \text { CE con nicotina } \\
\text { - CE sin nicotina }\end{array}$ & 3 semanas & $\begin{array}{l}\text { Reducción del } \\
\text { consumo al } 50 \%\end{array}$ & $\begin{array}{l}\text { CE con nicotina redujo } \\
\text { significativamente } \\
\text { cantidad de cpd }\end{array}$ \\
\hline Carpenter, 2017 & 68 individuos & $\begin{array}{l}\text { ECA } \\
2 \text { ramas, } 3 \text { semanas } \\
\text { - CE con nicotina } \\
\text { - control }\end{array}$ & 4 meses & $\begin{array}{l}\text { Reducción del } \\
\text { consumo } \\
\text { Abstinencia a los } 4 \\
\text { meses } \\
\text { Intentos de dejar de } \\
\text { fumar }\end{array}$ & $\begin{array}{l}\text { CE con nicotina redujo } \\
\text { significativamente la } \\
\text { cantidad de cpd; no } \\
\text { mostró diferencias en } \\
\text { tasas de abstinencia o } \\
\text { número de intentos }\end{array}$ \\
\hline Halpbern, 2018 & $\begin{array}{l}6.006 \text { individuos, no } \\
\text { seleccionados de } \\
\text { acuerdo a motivación } \\
\text { para dejar }\end{array}$ & $\begin{array}{l}\text { ECA, pragmático. } \\
\text { Participantes tenían } \\
\text { posibilidad gratuita a } \\
\text { acceder a uno de } \\
\text { estos: } 4 \text { ramas } \\
\text { - TRN en varias formas } \\
\text { - CE } \\
\text { - Incentivo económico } \\
\text { en voucher } \\
\text { - Incentivo económico } \\
\text { en depósito }\end{array}$ & 6 meses & $\begin{array}{l}\text { Abstinencia a los } 6 \\
\text { meses }\end{array}$ & $\begin{array}{l}\text { Los incentivos } \\
\text { económicos fueron } \\
\text { superiores a otras } \\
\text { intervenciones } \\
\text { Ofrecer CE o TRN no } \\
\text { mostró beneficios }\end{array}$ \\
\hline Hayek, 2019 & $\begin{array}{l}886 \text { individuos que } \\
\text { asistían a servicios de } \\
\text { cesación de } \\
\text { tabaquismo }\end{array}$ & $\begin{array}{l}\text { ECA } \\
2 \text { ramas, } 12 \text { semanas } \\
\text { - CE con nicotina } \\
\text { - TRN a elección }\end{array}$ & 12 meses & $\begin{array}{l}\text { Abstinencia continua a } \\
\text { los } 12 \text { meses verificada } \\
\text { por } \mathrm{CO}\end{array}$ & $\begin{array}{l}\text { CE fue más efectivo } \\
\text { que TRN para dejar de } \\
\text { fumar, cuando se } \\
\text { acompaña de apoyo } \\
\text { conductual }\end{array}$ \\
\hline
\end{tabular}

CE: cigarrillo electrónico, CO: monóxido de carbono; cpd: cigarrillos por día; ECA: ensayo clínico aleatorizado; TRN: terapia de reemplazo nicotínico.

\section{Estrategia de reducción del daño}

Un lugar que se piensa podrían tener los CE en el abordaje de la cesación tabáquica es en la llamada estrategia de reducción del daño. El concepto "reducción o control de daños", como programa de intervención ante los problemas derivados del abuso de drogas, comenzó a utilizarse a fines de los años 80 como respuesta, especialmente ante la epidemia del VIH/SIDA entre usuarios de drogas intravenosas. La estrategia de reducción de daños tiene como objetivo reducir las consecuencias negativas del uso de sustancias, más que en promover la abstinencia ${ }^{(40)}$. En cesación de tabaquismo histórica- 
mente no se ha promovido ni aceptado esta estrategia, ya que existe evidencia de daño asociado a cualquier nivel y dosis de consumo e incluso de exposición pasiva al humo, por lo que todos los tratamientos basados en evidencia contenidos en pautas y guías establecen como objetivo la abstinencia total y no la reducción de consumo ${ }^{(41-43)}$. En algunos sectores de la comunidad científica internacional se ha arraigado el concepto de que los CE son menos dañinos que el tabaco ${ }^{(44,45)}$, por lo que podrían ser considerados como estrategia de reducción del daño en aquellos fumadores que no logran o no desean dejar de fumar. El concepto de que los CE son $95 \%$ más seguros para la salud que el continuar fumando, que ha sido recogido en diferentes artículos y revisiones, deriva de un estudio de Nutt y colaboradores ${ }^{(46)}$, publicado en 2014. El estudio del cual se obtiene esa cifra es una consulta a expertos, donde se les solicita que realicen un ranking de cuán dañinos son algunos productos que contienen nicotina tales como cigarrillos, cigarros, pipas, pipas de agua, parches de nicotina, CE, tabaco masticado, entre otros. Las dimensiones de daños que se les consultaba a los expertos variaba desde daños a la salud, a la familia, a la comunidad, al ambiente, pérdida de vínculos, peligros, entre otros. Del llenado de un cuestionario y confección de índices basados en las respuestas de dichos expertos se estableció que el cigarrillo tenía un puntaje de 100, y el CE casi 5 puntos de acuerdo a dicha metodología, lo que deriva en esa aseveración de ser $95 \%$ menos dañinos. El estudio no contó con ninguna medición objetiva de daños a la salud, y entre los que contestaron los cuestionarios se encuentran asesores de la industria del CE. Una revisión Cochrane sobre estrategias de reducción de daño en tabaquismo, publicada en 2016, investiga diferentes herramientas entre las que incluye los CE para intentar reducir el consumo de tabaco sin abandonarlo. Lindson-Hawley y colaboradores concluyen en esta revisión que no hay evidencia que sostenga que los productos o herramientas que reducen el consumo de tabaco en algún grado logren disminuir el daño a la salud ${ }^{(47)}$.

\section{Impacto del CE en la prevalencia del consumo de tabaco a nivel poblacional}

Una de las preocupaciones que tiene la comunidad científica ante el uso masivo de CE es el real impacto de este fenómeno sobre la prevalencia de consumo de tabaco. Si el CE efectivamente ayuda a dejar de fumar sería esperable que la prevalencia de tabaquismo descendiera. No obstante, otras hipótesis y hechos afectan ese supuesto. Muchos vapeadores continúan fumando, lo que se denomina uso dual, los exfumadores expuestos a un ambiente de acceso al CE estarían más expuestos a recaer y los jóvenes que nunca fumaron podrían encontrar en el CE una "puerta de acceso" a la adicción a la nicotina y luego convertirse en tabaquistas al resultarles atractivo y más permitido que el cigarrillo ${ }^{(48)}$. Por ejemplo, entre los jóvenes de Estados Unidos el uso de CE se asoció a una mayor proporción de jóvenes que pasan de experimentar cigarrillos a consumirlos en forma regular, lo que apoya esta teoría de la puerta de $\operatorname{acceso}^{(49)}$.

Luego de los dos primeros estudios aleatorizados que mostraron efectividad del CE para dejar de fumar, surgieron otros estudios denominados del "mundo real", con el objetivo de estudiar el impacto de estos dispositivos en las tendencias de consumo de tabaco fuera del escenario del ensayo controlado. En 2014, Brown y colaboradores ${ }^{(50)}$ realizaron un estudio de corte transversal encuestando 5.863 adultos en Inglaterra que hubieran fumado en el último año y que hubieran realizado un intento para dejar de fumar sin ninguna ayuda o tratamiento, usando CE, o comprando TRN en farmacia no prescrita por médico. Los fumadores que intentaron dejar de fumar usando $\mathrm{CE}$ reportaron haber logrado la abstinencia en un $20 \%$, mientras que a los que usaron TRN comprada en farmacia alcanzaron la cesación en 10\% (Odds ratio $[\mathrm{OR}]=2,23,95 \%$ intervalo de confianza $(\mathrm{IC})=$ 1,70-2,93. Los fumadores que lo intentaron sin ninguna ayuda lo lograron en 15,4\%. Los autores concluyen que, entre fumadores que no busquen ayuda profesional para cesar, los CE son más efectivos que la TRN o la no ayuda. Este estudio también pone en evidencia cómo terapias con evidencia sólida de eficacia en los ensayos aleatorizados pierden efectividad en el mundo real debido a otras variables que entran en juego, como correcto uso, adherencia, adecuación de dosis, entre otros.

En 2016, el mismo grupo de autores, liderado esta vez por Beard, realizan otro estudio con el objetivo de estimar en qué medida la creciente prevalencia de uso de CE en Inglaterra se asocia con el éxito en dejar de fumar, la cantidad de intentos y el uso de medicaciones aprobadas para este uso o las consultas especializadas. Para ello, encuestaron en forma sistemática en repetidos cortes transversales a individuos mayores de 16 años entre 2006 y $2015^{(51)}$. Luego de ajustar por varios posibles factores de confusión, los autores encuentran que la tasa de éxito en un intento de cesación aumenta $0,098 \%$ por cada $1 \%$ de aumento de la prevalencia de uso de CE, concluyendo que el uso de $\mathrm{CE}$ ha incidido positivamente en el éxito de los intentos de dejar de fumar sin ayuda profesional. También reportan un menor uso de otras ayudas (fármacos de venta libre) y consultas especializadas desde la aparición del $\mathrm{CE}^{(51)}$.

No obstante, otros autores que observaron el comportamiento de los fumadores que usaron CE en el mun- 


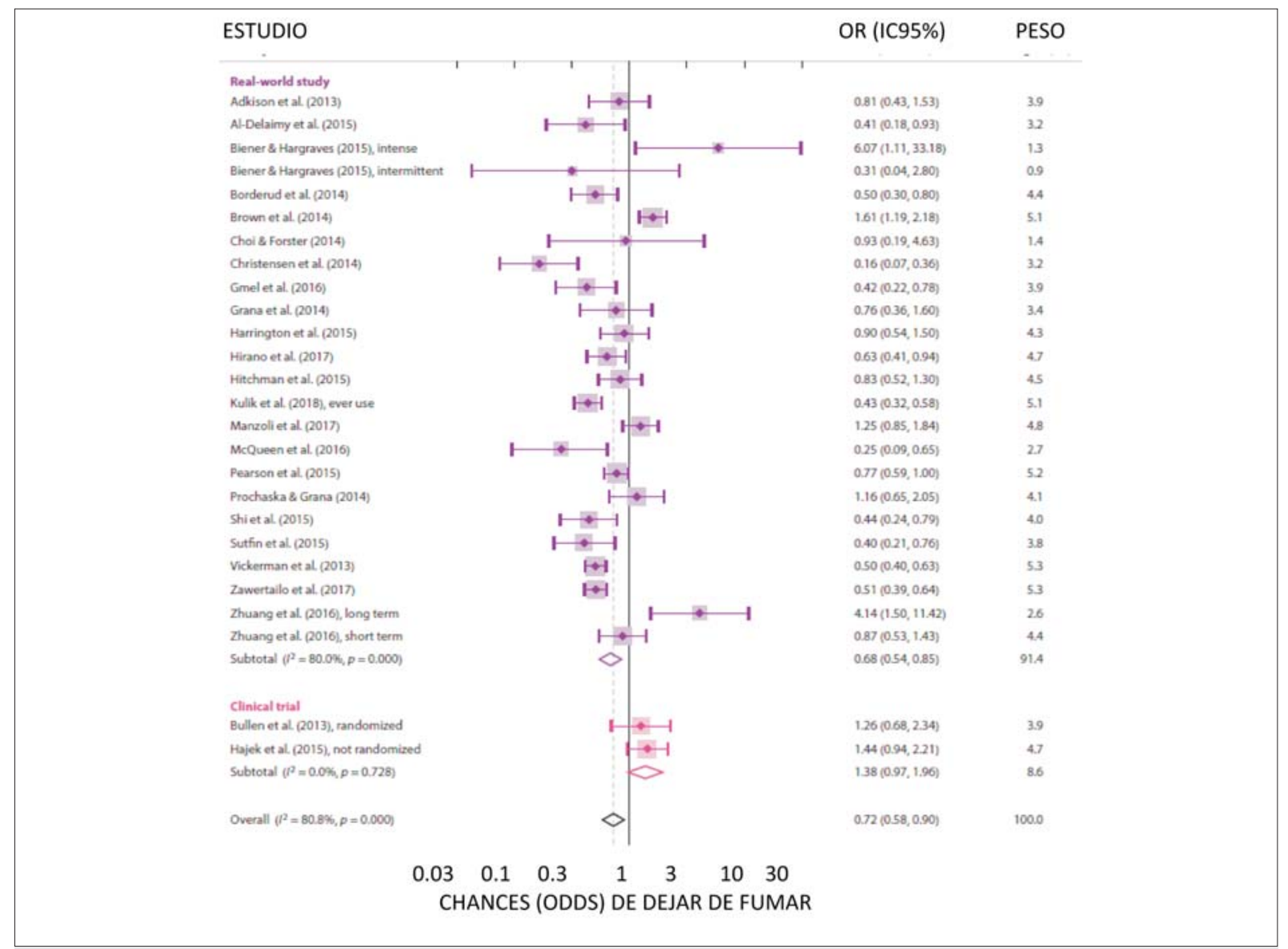

Figura 6. Diagrama forest plot que resume las chances de dejar de fumar disminuidas en $27 \%$ en quienes usan CE de acuerdo a los estudios analizados. Reproducida con permiso de Glantz y colaboradores ${ }^{(26)}$. IC: intervalos de confianza; OR: odds ratio

do real, reportan resultados contrarios a estos. Christensen y colaboradores, en 2014, publicaron un estudio basado en una encuesta a 9.656 adultos en el estado de Kansas, en Estados Unidos. Los autores encontraron que el uso de CE se asociaba con la realización de un intento para dejar de fumar; no obstante, se asociaba negativamente con lograr la abstinencia tabáquica, con significancia estadística ${ }^{(52)}$. Similares hallazgos encuentran autores como Al-Delaimy y Sutfin en estudios realizados en miles de usuarios en Estados Unidos ${ }^{(53,54)}$.

¿Cómo se interpreta entonces esta evidencia contradictoria y cómo se dirime la controversia? La realización de revisiones sistemáticas y metaanálisis que incluyan todos los estudios con resultados opuestos es la herramienta que puede contribuir a la interpretación de los datos. En ese sentido, Kalkhoran y Glantz realizan este análisis, incluyendo los estudios mencionados previamente y otros, totalizando datos de 38 estudios de buena calidad metodológica, de un total de más de 500 identificados. Los autores utilizaron una metodología que les permitió tanto analizar los estudios clínicos aleatorizados como las encuestas de uso de CE en el mundo real. Asimismo, analizaron los hallazgos de acuerdo a si el uso de CE se daba en fumadores intentando dejar de fumar o no. Los resultados son contundentes en mostrar que tal como están siendo utilizados, los $\mathrm{CE}$ se asocian con menores posibilidades de dejar de fumar ${ }^{(55)}$. Dos años más tarde, Glantz y Bareham vuelven a realizar un metaanálisis, agregando siete estudios más surgidos entre 2015 y 2017 , que no cambiaron sustancialmente el resultado, encontrando un OR: 0,73 IC95\% 0,59-0,92; lo que se traduce como que utilizar $\mathrm{CE}$ disminuye en $27 \%$ las chances de que un fumador deje de fumar (figura 6, reproducida con permiso del autor ${ }^{(26)}$ ).

Dos estudios recientes, publicados en 2019 y por lo tanto posteriores a los dos metaanálisis de Kalkhoran, Glantz y Bareham, merecen ser comentados. Recientemente, Soule y colaboradores ${ }^{(56)}$ publicaron un análisis longitudinal sobre las razones por las que utilizan los CE reportadas por los usuarios y los cambios en el estatus ta- 
báquico. Los datos proceden del estudio PATH (Population Assessment of Tobacco and Health), cohorte de Estados Unidos de 3.044 de participantes. El estudio realizó un seguimiento entre 2013 y 2015 con dos "olas" de recolección de datos de fumadores y exfumadores que utilizaban CE. Entre las olas 1 y 2, 27,2\% de los exfumadores se convirtieron en fumadores, mientras que $11,6 \%$ de los fumadores dejaron de fumar. El otro estudio, realizado en el Reino Unido por Hallinberg y colaboradores ${ }^{(57)}$, buscando esclarecer si el CE ha tenido impacto en la renormalización del fumar entre jóvenes y en las tendencias en la prevalencia de tabaquismo. Para ello, obtuvieron datos de 248.324 de jóvenes entre 13 y 15 años de las encuestas nacionales entre 1998 y 2015. Los autores concluyen que la aparición del CE no renormalizó el fumar, ya que las actitudes, creencias y conocimientos de los jóvenes acerca del tabaco no han cambiado luego de la aparición del CE. No obstante, sí encuentran un enlentecimiento significativo en la baja de la prevalencia de consumo de tabaco que se venía observando desde hace años, desde que entró en escena el CE.

Un aspecto no menor a tener cuenta frente a este tema controversial es que aproximadamente un tercio de las publicaciones no se ajusta a los requisitos de manifestar con claridad los conflictos de interés, en especial aquellas que aconsejan la utilización del CE. Es así que los artículos de autores con algún vínculo declarado que potencialmente genere conflicto de interés, muestran más del doble de posibilidades de presentar resultados favorables al $\mathrm{CE}^{(58,59)}$.

\section{Normativa nacional e internacional}

La introducción de los dispositivos electrónicos ha tenido una expansión en su consumo, especialmente en determinados mercados, lo cual ha permitido evaluar en esos países el impacto sobre la aplicación de las medidas sugeridas por el Convenio Marco para el Control del Tabaco. En la Conferencia de las Partes (COP 7), realizada en Nueva Delhi en el año 2016, se presentó un informe elaborado por el grupo de expertos de la OMS, en la cual se adopta la siguiente resolución: "La COP7 adoptó la decisión FCTC/COP7 en la que se invitaba a las Partes a que consideraran la posibilidad de aplicar algunas medidas de reglamentación propuestas en el informe preparado por la OMS en el documento FCTC/COP/7/11"(1). Entre las medidas se incluían la prohibición o restricción de la fabricación, importación, distribución, presentación, venta y uso de SEAN/SESN, según correspondiera a la legislación de las Partes y sus objetivos en materia de salud pública. Asimismo, se pidió a la secretaría del convenio que invitara a las Partes a que vigilaran los progresos científicos, normativos y de mercado -por ejemplo, en relación con la iniciación en el consumo de tabaco, su abandono, la publicidad y la promoción-e informaran al respecto, y se pidió a la OMS que informara sobre el desarrollo de métodos establecidos por organizaciones de normalización regionales e internacionales para el análisis y la medición de los contenidos y las emisiones de estos productos ${ }^{(39,60)}$. Según los datos de los informes presentados se ha producido un notorio incremento de la venta de estos productos, particularmente en algunos países. Los SEAN alcanzaron cifras de ventas de US\$ 2.760 millones en el año 2014 , pasando a US\$ 8.810 millones en 2016 y se estima que alcance los US\$ 26.840 millones en el 2023, en todo el mundo ${ }^{(61)}$. Los alcances de la reglamentación de los SEAN en los diferentes países se relaciona con la normativa vigente en cada uno de ellos, permitiendo clasificar las medidas adoptadas de la siguiente manera: 1) productos de tabaco; 2) productos que imitan el tabaco; 3) productos medicinales; 4) productos farmacéuticos; 5) productos de consumo; 6) sustancias tóxicas. En cuanto a los mecanismos de reglamentación se observan diferentes procedimientos: 1) algunos países han establecido nuevas leyes, decretos o resoluciones o algún otro mecanismo jurídico específico para reglamentar los SEAN; 2) otros utilizan legislación ya existente que puede aplicarse si la clasificación de los SEAN se ajusta al marco jurídico existente; 3 ) otros países han enmendado leyes existentes para incorporar los SEAN, y 4) algunos países utilizan una combinación de las medidas anteriores ${ }^{(62)}$. Según el informe de la OMS presentado para la COP 8 , se encuentran disponibles en 102 países de 181, de los cuales el $38 \%$ no ha reglamentado su utilización (tablas 2 y 3 ). Uruguay se encuentra dentro de los países en donde se ejerce algún tipo de prohibición de los SEAN. En nuestro país los CE y similares han sido regulados mediante dos decretos: 534/2009 y 299/2017 $7^{(63,64)}$. En el decreto 299/2017 se hacen extensivas las prohibiciones para el uso de CE y similares, a lo dispuesto por la Ley 18.256 para los productos del tabaco, por lo que se prohíbe su uso en lugares públicos cerrados. Uruguay se encuentra dentro de los países que posee una normativa estricta en lo referente a las prohibiciones para el uso de los productos de tabaco. Los dispositivos electrónicos se encuentran comprendidos dentro de estas restricciones, según lo expresan ambos decretos.

\section{Conclusiones e implicancias}

De acuerdo a la evidencia disponible al día de hoy sobre CE y otros dispositivos con referencia a sus componentes, se puede concluir que existen riesgos para la salud a corto y largo plazo para el consumidor, así como para los que inhalan vapor en forma pasiva. Tampoco se ha demostrado fehacientemente que sea un método efectivo para contribuir en la cesación de tabaquismo, pu- 
Tabla 2. Resumen de respuestas de las Partes (países) sobre los SEAN de acuerdo a lo informado al Convenio Marco para el Control del Tabaco de la OMS en 2018.

\begin{tabular}{|c|c|c|c|c|}
\hline \multirow[t]{3}{*}{ Región } & \multirow{3}{*}{$\begin{array}{l}\text { Número de Partes } \\
\text { por región }\end{array}$} & \multicolumn{3}{|c|}{ SEAN disponibles en el mercado nacional } \\
\hline & & \multirow{2}{*}{$\begin{array}{c}\text { Partes que respondieron } \\
\text { dónde hay SEAN } \\
\text { disponibles }\end{array}$} & \multicolumn{2}{|c|}{$\begin{array}{l}\text { Partes en las que hay SEAN disponibles pero no } \\
\text { están reglamentados }\end{array}$} \\
\hline & & & Número & Porcentaje \\
\hline África & 44 & 21 & 12 & $57 \%$ \\
\hline Américas & 30 & 17 & 6 & $35 \%$ \\
\hline Mediterráneo Oriental & 19 & 8 & 4 & $50 \%$ \\
\hline Europa & 51 & 38 & 7 & $18 \%$ \\
\hline Asia Sudoriental & 10 & 5 & 4 & $80 \%$ \\
\hline Pacífico Occidental & 27 & 13 & 6 & $46 \%$ \\
\hline Total & 181 & 102 & 39 & $38 \%$ \\
\hline
\end{tabular}

SEAN: sistemas electrónicos de administración de nicotina.

Tabla 3. Partes (países) en que los SEAN están prohibidos por Región de acuerdo a lo informado al Convenio Marco para el Control del Tabaco de la OMS en 2018.

\begin{tabular}{|c|c|c|c|c|c|c|}
\hline $\begin{array}{c}\text { Región } \\
\text { (Número de Partes } \\
\text { por Región) }\end{array}$ & $\begin{array}{c}\text { Europa } \\
\text { (Total: } 50 \text { Partes) }\end{array}$ & $\begin{array}{c}\text { África } \\
\text { (Total: } 44 \text { Partes) }\end{array}$ & $\begin{array}{l}\text { Pacífico Occidental } \\
\text { (Total: } 27 \text { Partes) }\end{array}$ & $\begin{array}{l}\text { Asia Sudoriental } \\
\text { (Total: } 10 \text { Partes) }\end{array}$ & $\begin{array}{c}\text { Américas } \\
\text { (Total: } 30 \text { Partes) }\end{array}$ & $\begin{array}{c}\text { Mediterráneo } \\
\text { Oriental } \\
\text { (Total: } 19 \text { Partes) }\end{array}$ \\
\hline $\begin{array}{l}\text { Partes en que los } \\
\text { SEAN están } \\
\text { prohibidos }\end{array}$ & Turkmenistán & $\begin{array}{l}\text { Etiopía } \\
\text { Mauricio } \\
\text { Uganda }\end{array}$ & $\begin{array}{l}\text { Australia } \\
\text { Brunéi } \\
\text { Camboya } \\
\text { Singapur }\end{array}$ & $\begin{array}{l}\text { Corea (República } \\
\text { Popular } \\
\text { Democrática de) } \\
\text { Nepal } \\
\text { Sri Lanka } \\
\text { Tailandia } \\
\text { Timor-Leste }\end{array}$ & $\begin{array}{l}\text { Brasil } \\
\text { México } \\
\text { Panamá } \\
\text { Suriname } \\
\text { Uruguay } \\
\text { Venezuela }\end{array}$ & $\begin{array}{l}\text { Arabia Saudita } \\
\text { Baréin } \\
\text { Egipto } \\
\text { Emiratos Árabes } \\
\text { Unidos } \\
\text { Irán } \\
\text { Jordania } \\
\text { Kuwait } \\
\text { Líbano } \\
\text { Omán } \\
\text { Catar } \\
\text { Siria }\end{array}$ \\
\hline
\end{tabular}

SEAN: sistemas electrónicos de administración de nicotina. 
diendo perpetuar el consumo dual de CE y convencional, manteniendo la adicción por la nicotina, así como iniciar el consumo en jóvenes y favorecer la recaída de exfumadores. Los profesionales de la salud deben asesorar a los consumidores, respetando los aspectos normativos y regulatorios que estos productos tienen en nuestro país, que buscan desalentar y desnormalizar el consumo de sustancias a través de estos dispositivos. Dada la creciente investigación clínica y epidemiológica sobre este tema, se deberá estar alerta a futuros estudios y evidencias que aporten más luz a la controversia.

\section{Agradecimientos}

A la Lic. Claudia Speranza de la Biblioteca del Sindicato Médico del Uruguay, por colaborar con la realización de la búsqueda bibliográfica.

Al Prof. Dr. Stanton Glantz de la Universidad de California, San Francisco, por permitirnos el uso de una figura de un artículo suyo.

\section{Abstract}

Introduction: e-cigarettes are systems that produce and release aerosol that contains nicotine and other substances. They have been controversial in the scientific community that works on tobacco control ever since they were created. There is evidence suggesting they are an alternative therapy for tobacco cessation and other studies prove they are harmful to health, and they end up being the door that welcomes young people to the world of nicotine addiction.

Objective: to collect evidence on e-cigarette in connection with: components, risks to the health, tobacco cessation, impact on the population and normative and regulatory aspects.

Method: Review of the literature. Bibliographic search on PubMed, MEDLINE, SCOPUS, Redalyc, Scielo, LiLACS, Dialnet, ScienceDirect, as well as in other documents of the World Health Organization, scientific societies, websites dedicated to consumers and the national and international laws.

Results: 130 documents were reviewed, and the 63 most relevant ones served as the grounds for the study. There is scientific evidence on the toxic and carcinogenic content of liquids and aerosols of e-cigarettes, as well as on their negative effect on cardiovascular and respiratory health. We present controversial studies on its effectiveness to give up smoking, evidence on how e-cigarettes have a negative influence on the initiation of consumption by the young and on ex-smokers relapse. The study also analyses the national and international norms regulating e-cigarettes.
Conclusions: health professionals must not recommend the use of e-cigarettes, since they are not safe and expose consumers and the general population to its direct and indirect harmful effects.

\section{Resumo}

Introdução: os cigarros eletrônicos $(\mathrm{CE})$ são sistemas que liberam aerossol contendo nicotina e outras substâncias. Desde seu surgimento geraram uma grande controvérsia na comunidade científica dedicada ao controle do tabaco, existindo evidências que os colocam como uma alternativa de tratamento para a cessação do tabagismo e outros estudos que mostram seus danos à saúde, e como se transformam na porta de entrada dos jovens a adição a nicotina.

Objetivo: recuperar a evidência disponível sobre CE com referência a: componentes, riscos para a saúde, cessação de tabagismo, impacto populacional e aspectos normativos e regulamentares.

Método: revisão narrativa. Fez-se uma pesquisa bibliográfica nas bases PubMed, MEDLINE, SCOPUS, Redalyc, Scielo, LiLACS, Dialnet, ScienceDirect, e também em publicações da Organização Mundial da Saúde, sociedades científicas, sites web dedicados a consumidores e legislação nacional e internacional.

Resultados: foram selecionados os 63 documentos mais relevantes dos 130 recuperados. Existem evidências científicas sobre conteúdos tóxicos e carcinogênicos em líquidos e aerossóis dos CE e também dos danos à saúde cardiovascular e respiratória. Apresentam-se estudos controversos sobre sua eficácia para apoiar o abandono do hábito de fumar e evidência de como os $\mathrm{CE}$ têm um impacto negativo na iniciação de jovens ao consumo e na recaída de ex- fumantes. Faz-se uma análise das normas nacionais e internacionais para regular os CE.

Conclusões: os profissionais de saúde não devem aconselhar o uso de CE por não ser seguros e por que expõem os consumidores e a população em geral aos efeitos deletérios diretos e indiretos de seu uso.

\section{Bibliografía}

1. World Health Organization. Conference of the Parties to the WHO Framework Convention on Tobacco Control. Electronic nicotine delivery systems and electronic non-nicotine delivery systems (ENDS/ENNDS). Report by WHO. 2016; (FCTC/COP/7/11, August 2016). Disponible en: https://www.who.int/fctc/cop/cop7/FCTC_COP_7_11_EN. pdf?ua=1\&ua=1 [Consulta: mayo 2019].

2. Centers for Disease Control and Prevention. Electronic cigarettes. 2018. Disponible en: https://www.cdc.gov/tobacco/basic_information/e-cigarettes/index.htm [Consulta: mayo 2019]. 
3. International Tobacco Control Policy Evaluation Project. ITC Uruguay national report. Findings from the wave 1 to 4 surveys (2006-2012). 2014. Disponible en: http://www.itcproject.org/files/ITC_Uruguay_Report-English-Sept24v24.pdf [Consulta: mayo 2019].

4. Llambí L, Parodi C, Barros M, Sevillano P, Pereira M, Sandberg M, et al. Cigarrillo electrónico y pipas de agua: conocimientos y uso entre estudiantes de Medicina, Uruguay, 2015. Rev Urug Med Int 2016; 1(3): 76-83.

5. Uruguay. Ministerio de Salud Pública. Encuesta Mundial de Tabaco en Adultos-Global Adult Tobacco Survey. 2017.

6. World Health Organization. Conference of the Parties to the WHO Framework Convention on Tobacco Control Electronic nicotine delivery systems. Report by WHO. 2014; (FCTC/COP/6/10, 21 July 2014). Disponible en: http://apps.who.int/gb/fctc/PDF/cop6/FCTC_COP6_10-en.p df [Consulta: mayo 2019].

7. Bam TS, Bellew W, Berezhnova I, Jackson-Morris A, Jones A, Latif E, et al. Position statement on electronic cigarettes or electronic nicotine delivery systems. Int J Tuberc Lung Dis 2014;18(1):5-7.

8. Álvarez R. Quién es JUUL y por qué los dueños de Philip Morris, fabricantes de Marlboro, están invirtiendo $12.800 \mathrm{mi}-$ llones en ella. 2019. Disponible en: www.xataka.com/medicina-y-salud/quien-juul-que-duenos-philip-morris-fabricantes-marlboro-estan-invirtiendo-12-800-millones [Consulta: mayo 2019].

9. Solovapeamos. Disponible en: http://www.solovapeamos.com/ [Consulta: mayo 2019].

10. Altet Gómez MN. Los cigarrillos electrónicos: “vapear”, un nuevo riesgo para los adolescentes (Revisión). Docplayer 2014. Disponible en: https://docplayer.es/12701933-Revision-los-cigarrillos-electronicos-vapear-un-nuevo-riesgo-para-los-adolescentes-maria-nieves-altet-gomez.html [Consulta: mayo 2019].

11. Palazzolo DL. Electronic cigarettes and vaping: a new challenge in clinical medicine and public health. A literature review. Front Public Health 2013; 1:56.

12. Orellana-Barrios MA, Payne D, Mulkey Z, Nugent K. Electronic cigarettes-a narrative review for clinicians. Am J Med 2015; 128(7):674-81.

13. Bertholon JF, Becquemin MH, Annesi-Maesano I, Dautzenberg B. Electronic cigarettes: a short review. Respiration 2013; 86(5):433-8.

14. Saffari A, Daher N, Ruprecht A, De Marco C, Pozzi P, Boffi R, et al. Particulate metals and organic compounds from electronic and tobacco-containing cigarettes: comparison of emission rates and secondhand exposure. Environ Sci Process Impacts. 2014; 16(10):2259-67.

15. Pisinger C. A systematic review of health effects of electronic cigarettes. 2015 (Document prepared for the World Health Organization, December 2015). Disponible en: https://www.who.int/tobacco/industry/product_regulation/BackgroundPapersENDS3_4November-.pdf?ua=1 [Consulta: mayo 2019].
16. Jiménez Ruiz CA, Solano Reina S, de Granda Orive JI, Signes-Costa Minaya J, de Higes Martinez E, Riesco Miranda JA, et al. El cigarrillo electrónico. Declaración oficial de la Sociedad Española de Neumología y Cirugía Torácica (SEPAR) sobre la eficacia, seguridad y regulación de los cigarrillos electrónicos. Arch Bronconeumol 2014; 50(8):362-7.

17. Soule EK, Maloney SF, Spindle TR, Rudy AK, Hiler MM, Cobb CO. Electronic cigarette use and indoor air quality in a natural setting. Tob Control 2017; 26(1):109-12.

18. Williams M, Villarreal A, Bozhilov K, Lin S, Talbot $\mathbf{P}$. Metal and silicate particles including nanoparticles are present in electronic cigarette cartomizer fluid and aerosol. PLoS One 2013; 8(3):e57987.

19. Lee WH, Ong SG, Zhou Y, Tian L, Bae HR, Baker N, et al. Modeling cardiovascular risks of e-cigarettes with human-induced pluripotent stem cell-derived endothelial cells. J Am Coll Cardiol 2019; 73(21):2722-37.

20. Vindhyal MR, Ndunda P, Munguti C, Vindhyal S, Okut H. Impact on cardiovascular outcomes among e-cigarette users: a review national health interview surveys. J Am Coll Cardiol 2019; 73(9 suppl 2):11.

21. Ndunda PM, Muutu TM. Electronic cigarette use is associated with a higher risk of stroke. Stroke 2019; (Suppl 1):A9.

22. Vardavas CI, Anagnostopoulos N, Kougias M, Evangelopoulou V, Connolly GN, Behrakis PK. Short-term pulmonary effects of using an electronic cigarette: impact on respiratory flow resistance, impedance, and exhaled nitric oxide. Chest 2012; 141(6):1400-6.

23. Li D, Sundar IK, Mcintosh S, Ossip DJ, Goniewicz ML, O'Connor RJ, et al. Association of smoking and electronic cigarette use with wheezing and related respiratory symptoms in adults: cross-sectional results from the Population Assessment of Tobacco and Health (PATH) study, wave 2. Tob Control 2019. doi: 10.1136/tobaccocontrol-2018-054694.

24. Moretto N, Volpi G, Pastore F, Facchinetti F. Acrolein effects in pulmonary cells: relevance to chronic obstructive pulmonary disease. Ann N Y Acad Sci 2012; 1259:39-46.

25. McCauley L, Markin C, Hosmer D. An unexpected consequence of electronic cigarette use. Chest 2012; 141(4):1110-3.

26. Glantz SA, Bareham DW. E-cigarettes use, effects on smoking, risks, and policy implications. Annu Rev Public Health 2018; 39:215-35.

27. U.S. Food \& Drug Administration. Some e-cigarette users are having seizures, most reports involving youth and young adults. 2019. Disponible en: https://www.fda.gov/tobacco-products/ctp-newsroom/some-e-cigarette-users-are-having-seizures-most-reports-involving-youth-and-young-adults [Consulta: mayo 2019]

28. Brownson EG, Thompson CM, Goldsberry S, Chong HJ, Friedrich JB, Pham TN, et al. Explosion injuries from E-Cigarettes. N Engl J Med 2016; 375:1400-2. doi:10.1056/NEJMc1608478. 
29. Burstyn I. Peering through the mist: systematic review of what the chemistry of contaminants in electronic cigarettes tells us about health risks. BMC Public Health 2014; 14:18.

30. Bullen C, Howe C, Laugesen M, McRobbie H, Parag V, Williman J, et al. Electronic cigarettes for smoking cessation: a randomised controlled trial. Lancet 2013; 382(9905):1629-37.

31. Caponnetto P, Campagna D, Cibella F, Morjaria JB, Caruso M, Russo C, et al. EffiCiency and Safety of an eLectronic cigAreTte (ECLAT) as tobacco cigarettes substitute: a prospective 12-month randomized control design study. PLoS One 2013; 8(6):1-12.

32. Hartmann-Boyce J, McRobbie H, Bullen C, Begh R, Stead LF, Hajek P. Electronic cigarettes for smoking cessation. Cochrane Database Syst Rev 2016; 9:CD010216.

33. Tseng TY, Ostroff JS, Campo A, Gerard M, Kirchner T, Rotrosen J, et al. A randomized trial comparing the effect of nicotine versus placebo electronic cigarettes on smoking reduction among young adult smokers. Nicotine Tob Res 2016; 18(10):1937-43.

34. Carpenter MJ, Heckman BW, Wahlquist AE, Wagener TL, Goniewicz ML, Gray KM, et al. A naturalistic, randomized pilot trial of E-cigarettes: uptake, exposure, and behavioral effects. Cancer Epidemiol Biomarkers Prev 2017; 26(12):1795-803

35. Halpern SD, Harhay MO, Saulsgiver K, Brophy C, Troxel AB, Volpp KG. A pragmatic trial of e-cigarettes, incentives, and drugs for smoking cessation. N Engl J Med 2018; 378(24):2302-10.

36. Hajek P, Phillips-Waller A, Przulj D, Pesola F, Myers Smith K, Bisal N, et al. A randomized trial of e-cigarettes versus nicotine-replacement therapy. N Engl J Med 2019; 380(7):629-37.

37. Bullen C, Verbiest M, Galea-Singer S, Kurdziel T, Laking G, Newcombe D, et al. The effectiveness and safety of combining varenicline with nicotine e-cigarettes for smoking cessation in people with mental illnesses and addictions: study protocol for a randomised-controlled trial. BMC Public Health 2018; 18(1):596.

38. Klonizakis M, Crank H, Gumber A, Brose LS. Smokers making a quit attempt using e-cigarettes with or without nicotine or prescription nicotine replacement therapy: impact on cardiovascular function (ISME-NRT)-a study protocol. BMC Public Health 2017; 17(1):293.

39. World Health Organization. Conference of the Parties to the WHO Framework Convention on Tobacco Control. Progress report on regulatory and market developments on electronic nicotine delivery systems (ENDS) and electronic non-nicotine delivery systems (ENNDS). Report by the Convention Secretariat. 2018; (FCTC/COP/8/10, 27 June 2018). Disponible en: https://www.who.int/fctc/cop/sessions/cop8/FCTC_COP_8_10-EN.pdf?ua=1 [Consulta: mayo 2019].
40. Velázquez Benítez D, Friman Rodríguez N, González García M. Programas de reducción de daños en las adicciones, un dilema ético. CCM 2016; 20(4):804-9.

41. Clinical Practice Guideline Treating Tobacco Use and Dependence 2008 Update Panel, Liaisons, and Staff. Treating tobacco use and dependence?: 2008 update. Rockville (MD): US Department of Health and Human Services; 2008.

42. Uruguay. Ministerio de Salud Pública. Programa Nacional para el Control del Tabaco. Guía nacional para el abordaje del tabaquismo Uruguay 2009. Montevideo: MSP, 2009.

43. U.S. Department of Health and Human Services, Centers for Disease Control and Prevention, National Center for Chronic Disease Prevention and Health Promotion (US) Office on Smoking and Health. The Health consequences of smoking-50 years of progress: a report of the Surgeon General. Atlanta, GA: CDC, 2004.

44. Mcneill A, Brose LS, Calder R, Hitchman SC, Hajek P, McRobbie H. E-cigarettes: an evidence update. A report commissioned by Public Health England. London: Public Health England, 2015. Disponible en: www.gov.uk/government/uploads/system/uploads/attachment_data/file/454516/Ecigarettes_an_evidence_update_A_report_commissioned_by_Public_Health_England.pdf [Consulta: mayo 2019].

45. Bauld L, McNeill A, Hajek P, Britton J, Dockrell M. E-cigarette use in public places: striking the right balance. Tob Control 2017; 26(e1):e5-6.

46. Nutt DJ, Phillips LD, Balfour D, Curran HV, Dockrell M, Foulds J, et al. Estimating the harms of nicotine-containing products using the MCDA approach. Eur Addict Res 2014; 20(5):218-25 .

47. Lindson-Hawley N, Hartmann-Boyce J, Fanshawe TR, Begh R, Farley A, Lancaster T. Interventions to reduce harm continued tobacco use. Cochrane Database Syst Rev 2016; (10): CD005231.

48. Feirman SP, Lock D, Cohen JE, Holtgrave DR, Li T. Flavored tobacco products in the United States: a systematic review assessing use and attitudes. Nicotine Tob Res 2016; 18(5):739-49.

49. Dutra LM, Glantz SA. Electronic cigarettes and conventional cigarette use among US adolescents: a cross-sectional study. JAMA Pediatr 2014; 168(7):610-7.

50. Brown J, Beard E, Kotz D, Michie S, West R. Real-world effectiveness of e-cigarettes when used to aid smoking cessation: a cross-sectional population study. Addiction 2014; 109(9):1531-40

51. Beard E, West R, Michie S, Brown J. Association between electronic cigarette use and changes in quit attempts, success of quit attempts, use of smoking cessation pharmacotherapy, and use of stop smoking services in England: time series analysis of population trends. BMJ 2016; 354:i4645.

52. Christensen T, Welsh E, Faseru B. Profile of e-cigarette use and its relationship with cigarette quit attempts and abstinence in Kansas adults. Prev Med 2014; 69:90-4 
53. Al-Delaimy WK, Myers MG, Leas EC, Strong DR, Hofstetter CR. E-cigarette use in the past and quitting behavior in the future: A population-based study. Am J Public Health 2015; 105(6):1213-9.

54. Sutfin EL, Reboussin BA, Debinski B, Wagoner KG, Spangler J, Wolfson M. The impact of trying electronic cigarettes on cigarette smoking by college students: a prospective analysis. Am J Public Health 2015; 105(8):e83-9.

55. Kalkhoran S, Glantz SA. E-cigarettes and smoking cessation in real-world and clinical settings: a systematic review and meta-analysis. Lancet Respir Med 2016; 4(2):116-28.

56. Soule EK, Plunk AD, Harrell PT, Hayes RB, Edwards KC. Longitudinal analysis of associations between reasons for electronic cigarette use and change in smoking status among adults in the Population Assessment of Tobacco and Health Study. Nicotine Tob Res 2019. doi: 10.1093/ntr/ ntz005.

57. Hallingberg B, Maynard OM, Bauld L, Brown R, Gray L, Lowthian E, et al. Have e-cigarettes renormalised or displaced youth smoking? Results of a segmented regression analysis of repeated cross sectional survey data in England, Scotland and Wales. Tob Control 2019; 1-10.

58. Pisinger C, Godtfredsen N, Bender AM. A conflict of interest is strongly associated with tobacco industry-favourable results, indicating no harm of e-cigarettes. Prev Med 2019; 119:124-31.

59. Martínez C, Fu M, Galán I, Pérez-Rios M, Martínez-Sánchez JM, López MJ, et al. Conflicts of interest in research on electronic cigarettes: a cross-sectional study. Tob Induc Dis 2018; 16:28. doi:10.18332/tid/90668.

60. Yoong SL, Stockings E, Chai LK, Tzelepis F, Wiggers J, Oldmeadow C, et al. Prevalence of electronic nicotine delivery systems (ENDS) use among youth globally: a systematic review and meta-analysis of country level data. Aust N Z J Public Health 2018; 42(3):303-8.

61. Research and marketing. Electronic cigarette market by product type, flavor and distribution channel - global opportunity analysis and industry forecast, 2017-2023. Disponible en: https://www.researchandmarkets.com/research/pjkd84/global_electronic?w=5 [Consulta: mayo 2019].

62. OMS. Conferencia de las Partes en el Convenio Marco de la OMS para el Control del Tabaco. Informe sobre los progresos normativos y de mercado en materia de sistemas electrónicos de administración de nicotina (SEAN) y sistemas electrónicos sin nicotina (SESN). Informe de la Secretaría del Convenio. 2018; (FCTC/COP/8/1, 27de junio de 2018).

63. Uruguay. Poder Ejecutivo. Decreto 534/009. Protección del derecho al medio ambiente libre de humo de tabaco y su consumo. Disponible en: https:/www.impo.com.uy/bases/decretos/534- 2009 [Consulta: mayo 2019].

64. Uruguay. Poder Ejecutivo. Decreto 299/017. Modificación del art. $1^{\circ}$ del decreto 534/009, relativo a la comercialización, importación, registro como marca o patente y publicidad de cualquier dispositivo electrónico para fumar. Disponible en: https://www.impo.com.uy/bases/decretos/299-2017 [Consulta: mayo 2019].

\section{Contribución de autores}

Todos los autores han participado de la concepción, diseño, recolección de datos, análisis redacción y revisión del manuscrito Laura Llambi, https://orcid.org/0000-0001-6636-792X.

Diego Rodriguez, https://orcid.org/0000-0002-7192-7255.

Carolina Parodi, https://orcid.org/0000-0002-5999-8018.

Enrique Soto, https://orcid.org/0000-0001-7831-4745. 